\title{
Ambiente Digital de Aprendizagem de Línguas com Apoio de Estruturas Digitais de Aprendizagem
}

\author{
Raissa Arruda de Araújo', Orivaldo de Lira Tavares ${ }^{1}$, Santinho Ferreira de Souza ${ }^{2}$ \\ ${ }^{1}$ Departamento de Informática e PPGI - CT - UFES \\ ${ }^{2}$ Departamento de Línguas e Letras - CCHN -UFES \\ \{raaraujo, tavares\}.inf.ufes.br, santinhodesouza@uol.com.br
}

Abstract. This paper presents a new digital environment for language learning ADALIN. In this environment, the teacher edits each learning activity in an EDA - Digital Learning Structs, with the level of proficiency to which it applies, the learning objective, the skills to be exercised, the description of the activity (what to do), the method (how to do the activity), and the digital resources (with what resources). An EDA is a specialized Pedagogical Architecture for language learning. Each EDA, when used by the learner, helps him understand what needs to be done and why it should be done, so he can build his knowledge. ADALIN is specially designed to practice the five important skills for learning foreign languages: listening, speaking, reading, writing and translation.

Resumo. Este artigo apresenta um novo ambiente digital para aprendizagem de línguas, o ADALIN. Nesse ambiente, o professor edita cada atividade de aprendizagem em uma EDA - Estrutura Digital de Aprendizagem, com o nível de proficiência ao qual se aplica, o objetivo de aprendizagem, as habilidades a serem exercitadas, a descrição da atividade (o que fazer), o método (como fazer a atividade) e os recursos digitais (com quais recursos). Uma EDA é uma Arquitetura Pedagógica especializada para a aprendizagem de línguas. Cada EDA, quando usada pelo aprendiz, ajuda-o a entender o que deve ser feito e o porquê deve ser feito, para que, assim, possa construir seu conhecimento. O ADALIN é especialmente projetado para exercitar as cinco habilidades importantes para a aprendizagem de línguas estrangeiras: audição, fala leitura, escrita e tradução.

\section{Introdução}

A evolução de tecnologias e meios de comunicação proporciona possibilidades de aprendizado em diversas áreas, e esse modo de organização e distribuição do conhecimento e do trabalho bem como de expectativas para com o novo implicam a necessidade de outras formas de pensar e de aproximação e fortalecimento das relações interpessoais. Configurado esse cenário, têm-se as línguas naturais como referência de observação, pois as pessoas, cada vez mais conectadas e com curiosidade para com o outro, constatam que aprender línguas favorece sua compreensão e inserção numa nova cultura.

Há iniciativas de produção de ambientes digitais de ensino-aprendizagem de línguas estrangeiras, os quais, a partir de diversas plataformas, por meio de conexão à 
internet, vêm potencializando a capacidade de aprendizado. Mas é preciso sempre pensar em inovações com qualidade. Nesse sentido, é esperada real disposição de professores e pesquisadores no desenvolvimento de tecnologias que estejam associadas à concepção diferenciada de ensino-aprendizagem de línguas, para que o aprendiz alcance nível de autonomia compatível com o contexto multilinguístico de envolvimento. São diversas as possibilidades, francamente favoráveis à organização de ambientes com gama variada de propostas pedagógicas, organização de conteúdo e avaliação dos resultados de aprendizagem.

Insere-se, nesse contexto, a proposição de ambiente de aprendizagem de línguas sistematizada em Arquiteturas Pedagógicas (AP) [Carvalho, 2005], especialmente projetadas, chamadas Estruturas Digitais de Aprendizagem (EDA), que permitem ao professor ter flexibilidade ao criar as atividades, de modo que o aprendiz possa exercitar, concomitantemente, as cinco habilidades necessárias para aprender uma nova língua.

As EDA são usadas em um Ambiente Digital de Aprendizagem de Línguas ADALIN, apresentado neste artigo, para promover a aprendizagem de línguas. O aprendiz pode selecionar uma EDA, para buscar atingir um certo objetivo de aprendizagem, ou pode solicitar a recomendação automática de uma EDA apropriada a seu nível de proficiência. Uma EDA, projetada para trabalho individual ou em grupo, é constituída pelos seguintes elementos: título, habilidades trabalhadas (uma ou várias), nível de proficiência, objetivo, atividades, métodos e recursos digitais.

Este artigo está organizado com as seguintes seções: 1. Introdução; 2. Fundamentação teórica e trabalhos correlatos, 3. Adalin, 4. Aplicação e Avaliação, e 5. Considerações Finais.

\section{Fundamentação teórica e trabalhos correlatos}

Esta seção apresenta o conceito de Arquitetura Pedagógica (AP), as cinco habilidades a serem exercitadas para a aprendizagem de línguas estrangeiras e a área de pesquisa de Computer Assisted Language Learning (CALL). Na sequência, apresenta-se alguns ambiente virtuais para aprendizagem de línguas referenciados em trabalhos científicos.

\subsection{Arquiteturas Pedagógicas}

Em um Ambiente Digital de Aprendizagem, é preciso que o aluno tenha mais autonomia e que o professor assuma papel de mediador. Nessa linha de pensamento, o ambiente descrito neste trabalho é baseado na criação e uso de Arquiteturas Pedagógicas (AP), um paradigma de ensino-aprendizagem baseada na junção de duas teorias pedagógicas muito conhecidas: o construtivismo, introduzido por [Piaget et al., 1978] a partir da década de 1940 e a autonomia, defendida por [Freire, 1989/2017].

O construtivismo é um modelo pedagógico clássico, introduzido por Jean Piaget que tem como base o equilíbrio entre os mecanismos de assimilação e acomodação. Na teoria Piagetiana, o conhecimento se dá e ocorre num desequilíbrio entre o que está acomodado e o diferente, iniciando-se um processo de assimilação, que parte da maturação de ideias, somado à interação com objetos e informações recebidas. De acordo com a teoria freiriana de autonomia [Freire, 1989/2017], aprender é um processo que acontece a partir do envolvimento do sujeito com o objeto de aprendizagem, numa tentativa de satisfazer sua curiosidade epistemológica. 
VII Congresso Brasileiro de Informática na Educação (CBIE 2018)

Anais dos Workshops do VII Congresso Brasileiro de Informática na Educação (WCBIE 2018)

AP são estruturas de aprendizagem organizadas a partir do encontro de componentes diferenciados como abordagem pedagógica, software, internet, inteligência artificial, educação a distância, concepção de tempo e espaço. Arquiteturas Pedagógicas são planejadas para atender aos anseios, experiências e reflexões dos envolvidos [Nevado et al. 2009]. Devem ser flexíveis e se adaptar a diversos enfoques temáticos, devem desenvolver mecanismos de autonomia de aprendizagem e pressupor que os aprendizes sejam protagonistas. Na literatura atual, são encontrados trabalhos onde se usam AP para promover aprendizagem como projetos de aprendizagem, debate de teses, entre outros. Neste trabalho uma AP, denominada Estrutura Digital de Aprendizagem (EDA), é usada para ser aplicada no ensino-aprendizagem de línguas. Uma EDA, além dos elementos comuns às diferentes AP (uma identificação, um objetivo de aprendizagem, as atividades a serem realizadas, método para realizar as atividades e um conjunto de recursos digitais), possui também a informação do nível de proficiência indicado para seu uso e as habilidades exercitadas durante o desenvolvimento da atividade proposta nela.

\subsection{CALL (Computer-Assisted Language Learning)}

O computador, enquanto máquina para aquisição de conhecimento, pode desempenhar papéis como repositório de informações, acompanhador do desenvolvimento, avaliador e mediador da aprendizagem individual ou em grupo, e meio de aproximação do aprendiz com a fonte de informação - quer seja ela um ser vivo, quer seja um objeto multimídia. Nesse contexto, CALL é um campo de pesquisa [Levy, 1997] definido pela aplicação de suporte computacional na aprendizagem e ensino de línguas. De acordo com [Warschauer, and Healey, 1998], vantagens como diversão, pares ou grupos pequenos trabalhando em conjunto, variedades de recursos disponíveis e estilos de aprendizado usados, exploração da aprendizagem com grandes quantidades de dados de línguas e várias formas de práticas com feedback são usados em CALL, de modo a promover a aquisição e melhoria de uma segunda língua. Além disso, [Dalia and Phil, 2018] abordam o computador como ferramenta de avaliação, desempenhando papel importante no ensino aprendizagem de línguas, uma vez que pode proporcionar o feedback imediato e privado, que fazem com que os aprendizes se motivem e sejam mais desinibidos e os ambientes de jogos voltados para o desenvolvimento linguístico, motivastes por usarem simulações de desafios do mundo real.

\subsection{Ambientes Digitais para Aprendizagem de Línguas}

A quantidade de tecnologias que podem ser usadas em CALL não é a única variável na escolha ou implementação de um ambiente com esse propósito. Exemplos de outras variáveis consideradas são: objetivos pedagógicos, decisões institucionais, curiosidades pessoais e tendências [Stockwell, 2007].

A plataforma Construtor de Arquiteturas Pedagógicas (CAP) [Reinoso et al, 2017] é uma plataforma digital que permite ao professor criar Arquiteturas Pedagógicas, sem que necessite programar recursos computacionais. As atividades criadas são bem definidas através da indicação de um título, um objetivo pedagógico, um conjunto de atividades a serem realizadas, um método para realizar as atividades e um conjunto de recursos digitais dentre aqueles disponíveis na biblioteca de recursos digitais (BRD) da plataforma CAP. Existe uma especialização da plataforma CAP, chamada CAP-APL (Tavares et al, 2017), em que os recursos digitais que compõem a BRD são apropriados para serem usados para a aprendizagem de Português e de Libras. 
VII Congresso Brasileiro de Informática na Educação (CBIE 2018)

Anais dos Workshops do VII Congresso Brasileiro de Informática na Educação (WCBIE 2018)

O Moodle, acrônimo para Modular Object-Oriented Dynamic Learning Environment, é uma plataforma digital de aprendizagem de código livre, amplamente usada por universidades de todo o mundo, acessível a partir de qualquer navegador web. Em [Wu and Hua, 2008], o Moodle é usado para mediar o aprendizado de inglês como língua estrangeira. Neste trabalho, o desenvolvimento de habilidades comunicativas na aprendizagem de línguas é baseado em um requisito: a interação social entre o professor e os alunos e entre os próprios alunos. As vantagens do desenvolvimento do curso na plataforma Moodle são que todas as interações são gravadas e o professor pode citá-las ou fazer interferências; os professores podem enviar mensagens para grupos de alunos; todos os participantes podem inscrever e receber mensagens a qualquer momento; a distância entre alunos e professores não é um desafio e é permitido o uso de diversas mídias. Porém concluíram que, apesar de ser uma ferramenta poderosa, não é um ambiente projetado para cursos de inglês, uma vez que não possui ferramentas apropriadas para o feedback dos estudantes, além de ser necessário o auxílio de um profissional de Tecnologia da Informação para usá-lo.

O ambiente American British Academy (ABA) pode ser acessado na web ou por dispositivos móveis. [Paiva, 2017] reporta-se ao ABA como um aplicativo móvel usado para aprendizagem da língua inglesa, com um método estrutural com gramática e tradução. O ABA usa recursos de vídeo, texto, áudio e atividades de repetição em conjunto com traduções. Nesse ambiente, é indicado que o método se baseia na aquisição natural da língua, em que primeiro se ouve, para aprender a falar e escrever e depois se entende a gramática da língua. $\mathrm{O}$ aprendiz pode se submeter a um teste para iniciar no nível apropriado. Este ambiente é freemium, isto é, apresenta partes gratuitas e outras pagas), assim apenas uma atividade de cada nível é disponibilizada para o acesso gratuito. Por fim, cada atividade é dividida na seguinte ordem: um pequeno filme, uma tarefa para verificação de pronúncia, uma tarefa para escrita, outra tarefa, uma vídeoaula, exercícios, vocabulário e uma avaliação, que só pode ser realizada após o aprendiz ter cumprido obrigatoriamente $100 \%$ das tarefas.

Duolingo [Munday, 2016] é um aplicativo educacional para aprendizagem de línguas gratuito cujas principais características são: o uso da técnica de memorização conhecida como "spaced repetition", intercalação de habilidades, feedback instantâneo e gamificação. Duas versões estão disponíveis para seus usuários: a versão para dispositivos móveis (móvel) e uma versão web. A versão móvel possui apenas as lições, ranking de pontuação entre os usuários e uma loja para a troca de recompensas ganhas através das lições. A versão web, com as mesmas funções que a móvel, possui uma lista de palavras aprendidas, um fórum de discussão e um sistema de tradução de textos colaborativo para usuários mais avançados. Para educadores, nesta mesma versão web, há um painel que permite a criação de grupos ou turmas, o acesso, de forma individual, às informações do aluno, em relação a lição (horário de realização, se está completa e a pontuação), e um fórum exclusivo destinado a problemas e ideias dos educadores. [Munday, 2016] reporta um estudo de caso em dois cursos de espanhol em uma universidade, usando o Duolingo como ferramenta complementar às aulas, com o intuito de reforçar o vocabulário e a gramática dos aprendizes, mas sem enfoque na competência comunicativa. Como resultado, os estudantes acharam fácil e preferem o uso do Duolingo a uma tarefa tradicional, utilizando livros, pela mobilidade e gamificação oferecida. O estudo não relata a opinião dos professores no uso do aplicativo. 
VII Congresso Brasileiro de Informática na Educação (CBIE 2018)

Anais dos Workshops do VII Congresso Brasileiro de Informática na Educação (WCBIE 2018)

\section{ADALIN}

O interesse de estudantes estrangeiros em aprender língua portuguesa, tanto para fins acadêmicos como para comunicação social, incentivou a oferta, sob a coordenação do Núcleo de Línguas da Universidade Federal do Espirito Santo, a oferecer um curso de português para estrangeiros, por meio um ambiente virtual de aprendizagem, tendo recaído a escolha no Moodle. Esse curso se tornou ponto de partida para uma pesquisa acadêmica envolvendo um projeto de pós-doutorado de Santinho de Souza e um projeto de mestrado em informática de Raissa Araújo, uma vez que despertou o interesse em pesquisas para a construção de ambiente digital mais apropriado para suporte a cursos para a aprendizagem de línguas.

Os requisitos do ADALin foram levantados por meio de uma série de reuniões entre desenvolvedores e usuários, em que se identificou a necessidade dos aprendizes exercitarem o uso efetivo da língua portuguesa e ao professor ter, como condição básica de trabalho, suporte de criação de AP em que se pudesse propor atividade composta do desenvolvimento em sequência variada das cinco habilidades - audição, fala, leitura, escrita e tradução. Nessa organização, uma EDA (Estrutura Digital de Aprendizagem) pode trabalhar com o desenvolvimento de uma ou mais habilidades, e, à medida que os textos vão sendo produzidos e reescritos, cada vez mais o aprendiz vai compreendendo como se dá o funcionamento da língua e suas práticas de uso nas diversas situações de fala e escrita.

Os níveis de proficiência configurados no ADALin se alinham à concepção do CELPE-Bras [Celpe-Bras 2018], certificado brasileiro de proficiência de língua portuguesa para estrangeiros. São eles: Intermediário, Intermediário Superior, Avançado, Avançado Superior. O conhecimento de língua considerado básico não tem certificação no CELPE-Bras.

\subsection{Protótipo do ADALin}

O protótipo do ADALin considera, com base nos requisitos levantados (Seção 3.3), prioritariamente, dois tipos de usuário: aprendiz e professor. Este ADA se concretiza em contextos operacionais modelados por Estruturas Digitais de Aprendizagem (EDA), que encapsulam as atividades propostas pelos professores e estão disponibilizadas para resolução pelos aprendizes. Concebidas, portanto, as atividades pelo princípio da autonomia, essas EDA possuem informações que permitem que os aprendizes tenham consciência do benefício de seu uso no processo de aprendizagem e se sintam estimulados à aprendizagem. Para a implementação, utilizou-se a linguagem de programação Python 3.5, que tem como características principais a interoperabilidade, é multi-plataforma, robusta, simples e de alto nível. A Python é usado para back-end junto com o sqlite através do framework Django, que possui facilidades com diversos pacotes, comunidade ativa, estrutura e simplicidade. No front-end, é usado a HTML5, em conjunto com o framework Bootstrap (CSS) e o framework Jquery. Algumas telas do protótipo implementado estão disponíveis em https://goo.gl/HvMioi .

As Estruturas Digitais de Aprendizagem (EDA) são formadas pelos seguintes elementos: título, objetivo de aprendizagem - o que se aprende ao usar a EDA), atividades - o que o aprendiz precisa fazer na EDA, método - como fazer as atividades propostas na EDA, habilidades - quais habilidades o aprendiz exercita na EDA, nível - 
VII Congresso Brasileiro de Informática na Educação (CBIE 2018)

Anais dos Workshops do VII Congresso Brasileiro de Informática na Educação (WCBIE 2018)

proficiência linguística necessária, e recursos digitais - recursos digitais que o aprendiz dispõe para desenvolver as atividades propostas na EDA.

\subsection{Arquitetura}

O Adalin foi desenvolvido com base na arquitetura padrão MVC (Models, Views e Controllers). Nesse modelo, o software é dividido em três camadas interconectadas. As EDA estão armazenadas nos Models, os modelos, são camadas de manipulação de dados: Views é a camada responsável pela interação do usuário; através das views são exibidos aos usuários os dados. A camada Controller é responsável por receber, enviar requisições do usuário e controlar as views, que são mostradas ao usuário.

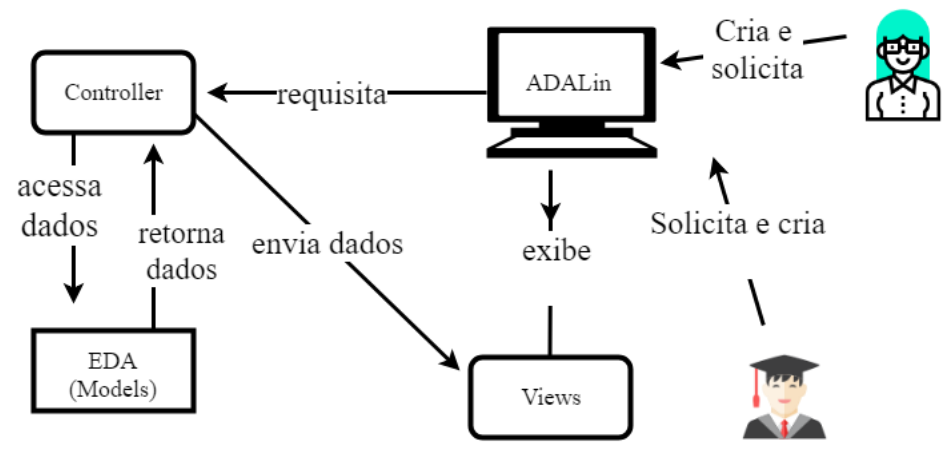

Figura 1 - Arquitetura MVC do ADALin

$\mathrm{Na}$ arquitetura do ADALin, o professor é responsável por criar as Estruturas Digitais de Aprendizagem, que ficam armazenadas na camada models; o aprendiz, por sua vez, acessa o ADALin, onde requisita as EDA disponíveis e cria soluções para elas.

\subsection{Requisitos do ADALin e Recursos Digitais}

Os requisitos do ADALin foram levantados com reuniões baseadas no método Joint Application Design (JAD) em que todos os participantes das reuniões são coautores da solução. Nesta seção, são apresentados os requisitos do ADALin, sendo que alguns deles já estão implementados no protótipo do ambiente.

O ambiente deve permitir que o professor possa:

- criar, editar e deletar EDA, assim como selecionar ou editar recursos digitais para as mesmas. Por questões de usabilidade, é importante que o ADA possa ser usado com recursos básicos de informática, não precisando assim, acessar códigos fontes, utilizar qualquer linguagem de marcação ou solicitar ajuda externa. Além de permitir que o professor possa usar os recursos de forma flexível, para construir atividades com todos os recursos que considerar necessários para desenvolvê-las, possibilita ao aprendiz exercitar as cinco habilidades isoladamente ou simultaneamente;

- acessar as soluções e percurso de aprendizagem dos aprendizes, além de permitir comentários sobre uma solução ou entrar em contato com o aprendiz.

O ambiente deve permitir ao aprendiz:

- usar uma ou várias EDA, quantas vezes considerar necessário, sendo que a escolha da EDA é feita pelo próprio aprendiz, que desenvolve seu percurso de aprendizagem de forma autônoma, a partir das informações que são 
VII Congresso Brasileiro de Informática na Educação (CBIE 2018)

Anais dos Workshops do VII Congresso Brasileiro de Informática na Educação (WCBIE 2018)

disponibilizadas. Além disso, deve permitir ao aprendiz solicitar recomendação de atividades mais apropriadas ao seu atual nível de proficiência;

- ter feedback imediato, em EDA que admita correção automática da solução;

- construir soluções em grupo para as EDA projetadas para soluções colaborativas;

- interagir com outros aprendizes e com professores.

Quanto aos requisitos não funcionais, o ambiente usa interface com o usuário, com design responsivo, permitindo que seja acessado por qualquer dispositivo que possua um navegador, facilitando que seja acessado em todas as plataformas. Por fim, os usuários devem estar autenticados no sistema, para que possam visualizar, inserir e editar dados.

Para o professor elaborar atividades que exercitem as cinco habilidades importantes à aprendizagem de línguas (audição, fala, leitura, escrita e tradução), o ADALin disponibiliza recursos digitais, de fácil configuração e combinação, de acordo com a necessidade do professor e do aprendiz. Para atividades individuais subjetivas, disponibiliza visualizador/editor de texto; visualizador/endereçador de vídeo externo; visualizador/gravador de vídeo; apresentador/gravador de áudio; editor/selecionador/visualizador de EDA; para atividades individuais objetivas: Recurso Quizzes para atividades objetivas individuais.

\subsection{Comparações entre ADA para Línguas}

A Tabela 1 apresenta uma comparação entre o ADALin e os trabalhos correlatos, apresentados na Seção 2.3.

Tabela 1 - Comparação entre Ambientes Digitais de Aprendizagem para Línguas

\begin{tabular}{|c|c|c|c|c|c|}
\hline & Duolingo & ABA & Moodle & CAP & ADALin \\
\hline Moldável e flexível para o professor & NÃO & NÂO & PARCIALMENTE & SIM & SIM \\
\hline $\begin{array}{l}\text { Possibilita exercitar as } 5 \text { habilidades } \\
\text { simultaneamente }\end{array}$ & NÃO & NÃO & NÃO & NÃO & SIM \\
\hline Especializado em línguas & SIM & SIM & NÃO & NÃO & SIM \\
\hline Gamificação & SIM & NÃO & NÃO & NÃO & NÃO \\
\hline Grava o percurso de aprendizagem & SIM & $S / D$ & NÃO & $S / D$ & SIM \\
\hline Usa arquiteturas pedagógicas & NÃO & NÃO & NÃO & SIM & SIM \\
\hline $\begin{array}{l}\text { Pode ser usar para aprendizagem que } \\
\text { não seja línguas }\end{array}$ & NÃO & NÃO & SIM & SIM & NÃO \\
\hline Recomenda atividades & SIM & NÃO & NÃO & NÃO & SIM \\
\hline $\begin{array}{l}\text { Criação de grupos para solucionar uma } \\
\text { atividade }\end{array}$ & NÃO & NÃO & SIM & NÃO & SIM \\
\hline Feedback Automatico (autorregulação) & SIM & SIM & NÃO & NÃO & $\begin{array}{l}\text { PARCIALME } \\
\text { NTE }\end{array}$ \\
\hline Incentivo à Interação & PARCIALMENTE & NÃO & PARCIALMENTE & NAO & SIM \\
\hline
\end{tabular}

Entre esses ambientes observa-se que: o Moodle, o CAP e o Adalin permitem ao professor criar atividades; o Duolingo e o ABA apenas disponibilizam lições já criadas; o ADALin, o Duolingo e o ABA dispõem de recursos internos que permitem exercitar as cinco habilidades; no Moodle, não é possível exercitar fala e audição sem o uso de recursos externos; o ADALin é o único em que o aprendiz pode realizar qualquer atividade ou solicitar a recomendação de atividade condizente com o seu perfil; o Moodle e o ADALin permitem a criação de grupos para trabalhar colaborativamente; o Duolingo e o ABA possuem feedback imediato em todas as atividades, enquanto o 
VII Congresso Brasileiro de Informática na Educação (CBIE 2018)

Anais dos Workshops do VII Congresso Brasileiro de Informática na Educação (WCBIE 2018)

ADALin oferece condições de feedback nas EDA individuais objetivas; o Moodle, em algumas atividades, e o CAP não apresenta essas condições.

Essa comparação entre os ambientes, destaca as novidades do ADALin em relação aos outros ambientes considerados.

\section{Aplicação e Avaliação}

Um protótipo do ADALin foi disposto para utilização e avaliação, tanto para professor como para aprendizes. Para realizarmos a avaliação do ambiente, dois questionários relacionados ao seu uso foram apresentados, um para o professor e outro para os aprendizes.

A avaliação foi realizada pelo professor responsável pelos cursos de Português como Língua Estrangeira da Universidade Federal do Espirito Santo, em ambiente controlado, onde se solicitou, a um professor dos cursos, a criação e configuração de EDA apropriada para ser usada pelos aprendizes. Após a criação da EDA, o professor respondeu este questionário foi preparado para professores que utilizem esse ambiente, disponível em https://goo.gl/t6k1Ms. O resultado mostra que o nível de dificuldade para criar uma EDA, no Adalin, foi considerado razoável, sendo que o avaliador considerou difícil "Descrever os elementos componentes da estrutura da atividade, levando em conta a concepção que ancora essa estrutura.". Ele definiu a diferença da criação de uma atividade encapsulada por uma EDA da seguinte forma: "uma EDA implica que haja sincronia entre atividade proposta e procedimentos de avaliação dos resultados coerente com essa atividade". Ele também considerou que os recursos oferecidos permitem trabalhar as cinco habilidades necessárias para aprendizagem de línguas.

A avaliação do ADALin realizada por aprendizes foi realizada em contexto de uso através do acesso ao endereço virtual do ambiente e ao questionário disponível em https://goo.gl/nNMbhW. Os participantes se cadastraram no ambiente, fizeram o uso das EDA disponíveis e responderam ao questionário. Os resultados obtidos são: dois terços dos aprendizes não sentiram dificuldade em usar a EDA, e os demais sentiram pouca dificuldade. Todos consideraram que a realização da atividade proposta na EDA leva a atingir o objetivo de aprendizagem previsto e a desenvolver as habilidades indicadas. Além disso, avaliaram que as informações disponíveis na EDA permitem a interpretação adequada da atividade e do método de solução proposto. Também avaliaram que as funções disponibilizadas foram suficientes para desenvolver a EDA.

\section{Considerações Finais}

Este artigo apresentou o ADALin no que se refere aos aspectos de sua arquitetura, a estrutura das EDA que podem ser criadas pelos professores e usadas pela comunidade de aprendizes, bem como a avaliação pelo professor e pelos aprendizes. O professor avaliou o ambiente quanto à facilidade e adequação para a criação de EDA. Os aprendizes avaliaram o uso de cada EDA e a facilidade e adequação do ambiente para desenvolverem as atividades propostas. Essas avaliações foram positivas, de modo geral, mas também indicaram sugestões de melhorias para versões futuras do ADALin.

Entre os requisitos levantados para a aprendizagem de língua, está o desenvolvimento de cinco habilidades: audição, fala, leitura, escrita e tradução. Para permitir a criação de atividades que desenvolvam essas habilidades, foram incluídos no 
VII Congresso Brasileiro de Informática na Educação (CBIE 2018)

Anais dos Workshops do VII Congresso Brasileiro de Informática na Educação (WCBIE 2018)

ADALin recursos digitais que operam sobre mídias para criação, edição e uso de textos escritos, textos sonoros e vídeos.

Os requisitos funcionais do ADALin foram apresentados, e outros requisitos foram especificados com o apoio de especialistas em ensino-aprendizagem de línguas: (i) as atividades estruturadas em EDA ficam disponibilizadas a qualquer aprendiz, apesar de serem construídas para aprendizes com certo nível de proficiência linguística, de modo a incentivar a curiosidade e aumentar a motivação em aprender; (ii) as interações e as atividades colaborativas ocorrem em uma comunidade de aprendizes, sem a separação por níveis de proficiência, para permitir a troca de experiências entre os mais adiantados e os neófitos, além de permitir a consolidação da aprendizagem, propiciada pelas situações de ensino-aprendizagem e aplicação do aprendido como resultado dessas interações entre sujeitos de diferentes modos de proficiência; (iii) as soluções construídas pelos aprendizes podem ser visitadas pelos colegas e comentadas, de modo a incentivar a proatividade, a aprendizagem colaborativa e o desenvolvimento do espírito reflexivo.

Atualmente está sendo ajustado o sistema de recomendação de EDA para cada aprendiz, com o uso dos níveis de proficiência exigidos em cada EDA, conforme indicação do professor responsável pela sua criação.

\section{Agradecimento}

O presente trabalho foi realizado com apoio da Coordenação de Aperfeiçoamento de Pessoal de Nível Superior - Brasil (CAPES) - Código de Financiamento 001.

\section{Referencias}

August, J. H. (1993). JAD, Joint Application Design. (Markon, Ed.). São Paulo.

Carvalho, M. J. S., Nevado, R. A. De, \& Menezes, C. S. De. (2005). Arquiteturas Pedagógicas para Educação a Distância: Concepções e Suporte Telemático, 351360.

Celpe-Bras (2018). Disponível em: $\quad<$ http://portal.inep.gov.br/acoesinternacionais/celpe-bras > . Acesso em Junho, 2018.

De Nevado, R. A., Dalpiaz, M. M., de Menezes, C. S., Nevado, R. A. de, Dalpiaz, M. M., \& Menezes, C. S. de. (2009). Arquitetura pedagógica para construção colaborativa de conceituações. In Anais do Workshop de Informática na Escola (Vol. 1, pp. 1653-1662).

Freire, P. (2017). A importância do ato de ler em três artigos que se completam (Vol. 22). Cortez editora.

Levy, M. (1997). Computer-assisted language learning: Context and conceptualization. Oxford University Press.

Moncada Linares, S., \& Díaz Romero, A. C. (2016). Developing a Multidimensional Checklist for Evaluating Language-Learning Websites Coherent with the Communicative Approach: A Path for the Knowing-How-to-Do Enhancement. Interdisciplinary Journal of E-Skills and Lifelong Learning, 12, 57-93. 
VII Congresso Brasileiro de Informática na Educação (CBIE 2018)

Anais dos Workshops do VII Congresso Brasileiro de Informática na Educação (WCBIE 2018)

Munday, P. (2016). The case for using DUOLINGO as part of the language classroom experience. RIED: Revista Iberoamericana de Educación a Distancia, 19(1), 83101.

Piaget, J., Amann, M., \& de Paula Leite, C. L. (1978). Fazer e compreender.

Reinoso, Luiz; Amorim, Marcello; Tavares, Orivaldo; Almeida, Renan. Framework CAP 1.0 para criação e uso de arquiteturas pedagógicas. In: XXVIII Simpósio Brasileiro de Informática na Educação SBIE, 2017, Recife. Editora: Sociedade Brasileira de Computação, 2017.

Tavares, Orivaldo; Reinoso, Luiz; Almeida, Wanderson. CAP-APL: plataforma para criação e uso de arquiteturas pedagógicas para aprendizagem de Português e Libras. In: XXVIII Simpósio Brasileiro de Informática na Educação SBIE, 2017, Recife. Editora: Sociedade Brasileira de Computação, 2017.

Wu, W.-S., \& Hua, C. (2008). The application of Moodle on an EFL collegiate writing environment. Journal of Education and Foreign Languages and Literature, 7(1), $45-56$.

Paiva, V. L. M. de O. e. (2017). Aplicativos móveis para aprendizagem de língua inglesa, (6), 10-31.

Dalia, D., \& Phil, M. (2018). Technology CALLs You Out of the Ordinary !, 18(49042), 49-53.

Warschauer, M., \& D. Healey. (1998). Computers and language learning: An overview. Language Teaching, 31, pp. 57-71

Stockwell, G. (2007). A review of technology choice for teaching language skills and areas in the CALL literature. ReCALL, 19(02), 105. 